\title{
Fee waiver
}

Public Domain

\section{Source}

Open Research Glossary

If an institution, research funder or author cannot pay for an Article Processing Charge, many publishers or journals will offer partial or total waiving for fees. 